\title{
Wound Infection After Excision and Primary Midline Closure for Pilonidal Disease: Risk Factor Analysis to Improve Patient Selection
}

\author{
Sotirios Popeskou • Dimitrios Christoforidis • \\ Christiane Ruffieux • Nicolas Demartines
}

Published online: 8 October 2010

(C) Société Internationale de Chirurgie 2010

\begin{abstract}
Background Excision and primary midline closure for pilonidal disease (PD) is a simple procedure; however, it is frequently complicated by infection and prolonged healing. The aim of this study was to analyze risk factors for surgical site infection (SSI) in this context.

Methods All consecutive patients undergoing excision and primary closure for PD from January 2002 through October 2008 were retrospectively assessed. The end points were SSI, as defined by the Center for Disease Control, and time to healing. Univariable and multivariable risk factor analyses were performed.

Results One hundred thirty-one patients were included [97 men (74\%), median age $=24$ (range 15-66) years]. SSI occurred in $41(31 \%)$ patients. Median time to healing was 20 days (range 12-76) in patients without SSI and 62 days (range 20-176) in patients with SSI $(P<0.0001)$. In univariable and multivariable analyses, smoking $[\mathrm{OR}=2.6$ (95\% CI 1.02, 6.8), $P=0.046]$ and lack of antibiotic prophylaxis $[\mathrm{OR}=5.6(95 \%$ CI $2.5,14.3), P=0.001]$
\end{abstract}

Presented at the 4th Scientific and Annual Meeting of the European Society of Coloproctology (ESCP), Prague, 23-26 September 2009.

S. Popeskou · D. Christoforidis · N. Demartines

Division of Visceral Surgery, Department of Surgery,

CHUV, University of Lausanne, Lausanne, Switzerland

C. Ruffieux

Institute of Preventive and Social Medicine, Biostatistics,

University of Lausanne, Lausanne, Switzerland

D. Christoforidis $(\bowtie)$

Service de Chirurgie Viscérale, BH 15 CHUV,

1011 Lausanne, Switzerland

e-mail: christoforidis@chuv.ch were significant predictors for SSI. Adjusted for SSI, age over 25 was a significant predictor of prolonged healing. Conclusion This study suggests that the rate of SSI after excision and primary closure of PD is higher in smokers and could be reduced by antibiotic prophylaxis. SSI significantly prolongs healing time, particularly in patients over 25 years.

\section{Introduction}

Pilonidal disease (PD) is a common condition that affects a significant part of the young active population [1]. Despite its benign nature it may adversely impact physical capacity for long periods and is responsible for significant time off work with economical consequences. While acute pilonidal abscess is generally treated with incision and drainage, management of chronic or recurrent PD is still controversial. There is currently no single therapy that ideally combines a minimally invasive procedure with fast healing times, low recurrence rates, and good esthetic results. Surgical techniques vary in the extent of excision of PD (radical or minimal) and type of closure of the defect (primary closure at the midline, off the midline, with or without skin flaps, or no closure and open healing). A recent Cochrane review analyzed 18 randomized controlled trials and concluded that primary closure leads to faster healing times and the same surgical site infection (SSI) rates but more recurrences compared to open healing. The authors also concluded that off-midline closure leads to less SSI, faster healing times, and fewer recurrences compared to midline closure. However, nine patients would need to be treated by an off-midline procedure to prevent one surgical site infection and 11 would need to be treated to prevent one recurrence [2]. Midline or 
off-midline primary closure may turn out not to be the ideal option if SSI occurs as this will inevitably lead to wound breakdown and prolonged healing times. Therefore, SSI prevention is a major step in improving outcomes of PD management. Although several large studies have analyzed risk factors for SSI following a variety of surgical interventions, only one study has performed multivariable risk factor analysis for SSI in the specific context of PD treated by excision and primary closure [3]. Many surgeons are not familiar with the more complex primary closure techniques using flaps. Probably because of its simplicity, excision and midline closure is a widely applied technique for management of uncomplicated PD in many institutions, including our own over the past decade. A better understanding of the risk factors for SSI and the resulting wound-healing complications in this context would help select those patients who could benefit from this simple technique. The aim of this study was to perform a risk factor analysis for SSI after excision and primary midline closure for PD.

\section{Methods}

The study was approved by the local ethics committee.

All consecutive patients operated for PD in a teaching hospital from January 2002 through March 2008 were retrospectively analyzed. The primary patient list was generated electronically through diagnostic and surgical intervention codes. We included only patients with a documented follow-up who underwent excision and primary closure for $\mathrm{PD}$ and excluded patients who underwent incision and drainage only for acute pilonidal abscess or patients whose wounds were left completely or partially open for secondary healing. PD was classified according to Tezel et al. [4] (Table 1).

Surgery was performed under general anesthesia usually by a surgical resident under direct supervision of a consultant surgeon. A symmetric elliptical incision around midline pits in the navicular area was extended laterally as needed to include all lateral sinuses and to remove en bloc

Table 1 Pilonidal disease classification [4]

Type I: Asymptomatic pit(s) without a history of abscess and/or drainage

Type II: Acute pilonidal abscess

Type III: Pit(s) within the navicular area with a history of abscess and/or previous drainage

Type IV: Extensive disease where one or more sinus openings lie outside the navicular area

Type V: Recurrent pilonidal sinus following any surgical treatment all infected cutaneous and subcutaneous tissue. The defect was closed primarily with interrupted subcutaneous and skin sutures without any sort of flap, creating a midline scar with occasional lateral extensions according to the lateral sinus anatomy. Use of antibiotic prophylaxis (single dose given at anesthesia induction), methylene blue to identify sinuses, and suction drains were at the surgeon's discretion. Prior to 2007, most patients were admitted to the hospital postoperatively. Since then, the vast majority of interventions for PD are performed as day-surgery. Patients were followed at our outpatient wound clinic for dressing changes by specialized nurses and by a surgery resident under the supervision of a registrar until complete wound healing.

The primary end point of this study was SSI defined according to the Center for Disease Control (CDC) guidelines [5] (Table 2). The criteria for SSI diagnosis were recorded. The secondary end point was time to healing, defined as the number of days from surgery until suture removal for noncomplicated wounds or until no further need for wound dressings in dehiscent wounds.

Statistical analysis was performed using STATA version 10.1. Patients with SSI were compared to patients without SSI for potential predictive variables using the $\chi^{2}$ test for categorical variables and the Wilcoxon test for continuous variables. Age was taken as a continuous variable and also as a categorical variable with two categories $(<25$ years, $\geq 25$ years) based on the median value. Odds ratios with $95 \%$ confidence intervals were given as a measure of the effect size of each potentially predictive variable. A multivariable logistic regression model was built using a backward strategy. All variables with $P \leq 0.2$ in the univariable analysis were candidates for inclusion in the multivariable model. Interaction terms were tested. Only those variables with $P<0.05$ were retained in the final model. Goodness of fit was tested using the HosmerLemeshow test. Medians of time to healing were compared

Table 2 CDC guidelines for superficial surgical site infection

Infection occurs within 30 days after the operation and involves only skin or subcutaneous tissue of the incision and at least one of the following:

1. Purulent drainage, with or without laboratory confirmation, from the superficial incision

2. Organisms isolated from an aseptically obtained culture of fluid or tissue from the superficial incision

3. At least one of the following signs or symptoms of infection: pain or tenderness, localized swelling, redness, or heat and superficial incision is deliberately opened by surgeon, unless incision is culture-negative

4. Diagnosis of superficial incisional SSI by the surgeon or attending physician 
between patients for the same potential predictor variables using the Wilcoxon or the Kruskal-Wallis test. A multivariable ANOVA model was built. To cope with the heteroscedasticity, the logarithm of days to healing was taken as the dependent variable. The backward strategy was used, choosing $P \leq 0.2$ for a predictor to be potentially included in the model and $P<0.05$ for a predictor to be retained in the final model. Interaction terms were tested. Nonparametric post test was used to compare the healing time between age groups stratified by infection.

\section{Results}

Of 190 patients who underwent elective surgery for PD over the study period, 131 met inclusion criteria and were analyzed. We excluded 33 patients treated with secondary healing and 26 due to missing or incomplete medical records. Patient characteristics are given in Table 3.

A SSI was documented in $41(31 \%)$ patients (suture removal for drainage in 33 , abscess incision in 2 , antibiotic prescription for purulent drainage in 2 , and wound dehiscence with purulent drainage in another 4).

In univariable and multivariable analyses, smoking and no use of preoperative antibiotic prophylaxis were significantly associated with a higher risk of SSI (Tables 4, 5). The final logistic regression analysis model was not rejected by the test of goodness of fit $(P=0.4)$.

The overall median time to healing was 25 (range 12170) days. It was significantly shorter in the group without SSI [without SSI: 20 (12-76) days; with SSI: 62 (20-176) days, $P<0.0001]$. Healing took more than 1 month in 55 (42\%) patients, more than 2 months in 24 (18\%) patients, and more than 3 months in $8(6 \%)$ patients.

In univariable analysis, time to healing was significantly longer in patients who developed a SSI, in those who did

Table 3 Patient characteristics

\begin{tabular}{ll}
\hline Men, $n(\%)$ & $97(74)$ \\
Age (years), median (range) & $24(15-66)$ \\
Type of PD & I $8(6.1)$ \\
& II 0 (excluded) \\
& III $96(73.2)$ \\
& IV $12(9.1)$ \\
& V $14(10.6)$ \\
Smoking, $n(\%)$ & $77(59)$ \\
BMI (kg/m $\left.{ }^{2}\right)$, median (range) & $24.1(17.7-33.7)$ \\
Use of antibiotic prophylaxis, $n(\%)$ & $102(78)$ \\
Use of methylene blue, $n(\%)$ & $94(72)$ \\
Use of suction drain, $n(\%)$ & $81(62)$ \\
Hospital stay (days), median (range) & $2(0-11)$ \\
\hline
\end{tabular}

not receive antibiotic prophylaxis, and in those with a BMI of $25 \mathrm{~kg} / \mathrm{m}^{2}$ and over (Table 6). In multivariable analysis (using ANOVA model with log of time as dependent variable), patients with infection aged 25 years and older had a longer healing time than those under 25 (25 and older: 70.5 (27-170) days; under 25 years: 48 (20-115) days, $P=0.02$ for age and 0.03 for interaction).

\section{Discussion}

In this study we performed risk factor analysis for SSI on 131 patients treated for PD with excision and primary midline closure. The results suggest that the absence of antibiotic prophylaxis and smoking habits are independent predictors of SSI. We found a SSI rate of $33 \%$, which is in the upper range of reported SSI rates in this context $[6,7]$. In a pooled analysis of 35 studies that included 1731 patients treated with excision and primary midline closure for PD, the SSI rate was $12.4 \%$ (95\% CI 11.1-13.8; range $0-38.5 \%)$ [6].

PD falls in the category of "contaminated" or "dirty" surgical wounds and administration of antibiotic prophylaxis prior to surgery is therefore theoretically recommended [8]. During the study period, in the absence of institutional guidelines, use of antibiotic prophylaxis was left up to the surgeon's discretion and often omitted. Our results confirm the rationale to use antibiotic prophylaxis, but interestingly, the only randomized study performed in this setting challenges this recommendation. Sondenaa et al. [9, 10] randomized 153 patients with PD undergoing excision and primary closure to receive a single dose of $2 \mathrm{~g}$ cefoxitin intravenously or no prophylaxis at all. They found no difference in wound complication rates, time to healing, or recurrence rates. On the other hand, a recent randomized trial suggested that a broad-spectrum 5-day antibiotic regimen is superior to a single-dose antibiotic prophylaxis in preventing infection-related wound complications following excision and primary closure of PD [11]. Others failed to demonstrate a benefit with prolonged use of antibiotics $[3,12]$. In the absence of a definitive consensus on this subject, based on our present results we have introduced an institutional recommendation for a single-dose antibiotic prophylaxis in this context.

Besides antibiotic prophylaxis, tobacco smoking was the only other significant predictor of SSI in our multivariable analysis. Smoking has been identified as an independent risk factor for SSI in a variety of surgical procedures, including cardiac surgery [13], spine surgery [14], plastic surgery [15], and general and vascular surgery [16]. The proposed mechanisms of increased infection rates in smokers is a tobacco-induced decrease in tissue oxygenation [17], an increase in bacterial virulence, and a 
Table 4 Univariable analysis for SSI

\begin{tabular}{|c|c|c|c|c|c|c|}
\hline Variable & & no SSI $(n=90)$ & $\operatorname{SSI}(n=41)$ & OR & $95 \% \mathrm{CI}$ & $P$ value \\
\hline \multirow[t]{2}{*}{ Gender } & Women & $25(71.1)$ & $10(28.9)$ & 0.84 & $0.4,2.0$ & 0.685 \\
\hline & Men & $65(67.7)$ & $31(32.3)$ & & & \\
\hline \multirow[t]{3}{*}{ Age (years) } & Mean (SD) & $25.8(7.8)$ & $27.9(7.0)$ & & & 0.060 \\
\hline & $\geq 25$ & $42(65.6)$ & $22(34.4)$ & 1.3 & $0.6,2.8$ & 0.4 \\
\hline & $<25$ & $48(71.6)$ & $19(28.4)$ & & & \\
\hline $\operatorname{BMI}\left(\mathrm{kg} / \mathrm{m}^{2}\right)$ & Mean (SD) & $24.6(3.6)$ & $25.3(3.1)$ & & & 0.157 \\
\hline \multirow[t]{2}{*}{ Obesity (BMI $\geq 25 \mathrm{~kg} / \mathrm{m}^{2}$ ) } & Yes & $28(62.2)$ & $17(37.8)$ & 1.7 & $0.7,3.7$ & 0.194 \\
\hline & No & $53(73.6)$ & $19(26.4)$ & & & \\
\hline \multirow[t]{2}{*}{ Smoking } & Yes & $48(62.3)$ & $29(37.7)$ & 2.3 & $1.0,5.6$ & 0.050 \\
\hline & No & $35(79.5)$ & $9(20.5)$ & & & \\
\hline \multirow[t]{4}{*}{ Type of PD } & I & $6(75)$ & $2(25)$ & & & 0.959 \\
\hline & III & $67(69.1)$ & $30(30.9)$ & & & \\
\hline & IV & $8(66.7)$ & $4(33.3)$ & & & \\
\hline & V & $9(75)$ & $5(25)$ & & & \\
\hline \multirow[t]{2}{*}{ Use of antibiotic prophylaxis } & Yes & $79(77.5)$ & $23(22.5)$ & 0.18 & $0.07,0.4$ & $<0.0001$ \\
\hline & No & $11(37.9)$ & $18(62.1)$ & & & \\
\hline \multirow[t]{2}{*}{ Use of suction drain } & Yes & $59(72.8)$ & $22(27.2)$ & 0.61 & $0.3,1.3$ & 0.194 \\
\hline & No & $31(62)$ & $19(38)$ & & & \\
\hline \multirow[t]{2}{*}{ Use of methylene blue } & Yes & $67(71.3)$ & $27(28.7)$ & 0.66 & $0.3,1.5$ & 0.311 \\
\hline & No & $23(62.2)$ & $14(37.8)$ & & & \\
\hline
\end{tabular}

Results are shown as absolute patient numbers and percentage of SSI within the variable in parenthesis, unless otherwise indicated

Table 5 Multivariable analysis for SSI

\begin{tabular}{lll}
\hline Variable & Women & OR $(95 \% \mathrm{CI})$ \\
\hline Gender & Men & - \\
& $\geq 25$ & - \\
Age (years) & $<25$ & \\
Obesity (BMI $\left.\geq 25 \mathrm{~kg} / \mathrm{m}^{2}\right)$ & Yes & - \\
& No & \\
Smoking & Yes & $2.59(1.01,6.62)$ \\
& No & \\
Type of PD & 1 & - \\
& 3 & \\
& 4 & \\
Use of antibiotic prophylaxis & Yes & $0.16(0.06,0.41)$ \\
Use of suction drain & No & \\
Use of methylene blue & Yes & - \\
& No & \\
& Yes & - \\
& No & \\
& &
\end{tabular}

dysregulation of immune function [18]. It is likely that the anoperineal area is particularly sensitive to the adverse effects of tobacco, as illustrated by the strong association of smoking with hidradenitis suppurativa [19].
Al-Khayat et al. [3] published the only other study with multivariable risk factor analysis for SSI after excision and primary closure of PD. In their study, all 94 patients received antibiotic prophylaxis for at least 24 hours and underwent offmidline Karydakis closure. The overall SSI rate was $12.8 \%$. The authors found tobacco smoking and obesity to be the only independent factors of SSI. Obesity is another well-recognized risk factor for SSI [20]. In our study, obese patients seemed to suffer SSI more often but the difference was not significant. Obesity seemed to delay time to healing upon univariable analysis but this was not confirmed after adjusting for confounding factors in multivariable analysis, possibly because of the relatively small sample size of our study.

Factors related to the surgical technique, such as use of a suction drain and methylene blue, were not found to be significant predictors of SSI. In two small randomized studies [21, 22], the use of drains seemed to be deleterious after excision and Limberg flap closure of PD, but in another small randomized study [23] they were beneficial after Karydakis flap closure. The impact of methylene blue use on SSI is unknown, but it was shown to reduce the recurrence rate significantly [24]. Subcutaneous sutures were placed in all our study patients to close the wound, so therefore this factor could not be analyzed. In a randomized study, however, no significant difference in SSI rates was found after excision and primary closure of PD with or without subcutaneous sutures [25]. 
Table 6 Univariable analysis for time to healing
* Wilcoxon and Kruskall-Wallis tests

\begin{tabular}{|c|c|c|c|c|}
\hline Variable & & Mean (SD) & Median (range) & $P$ value \\
\hline \multirow[t]{2}{*}{ SSI } & No & $23.2(11.4)$ & $20(12 ; 76)$ & $<0.0001$ \\
\hline & Yes & $67.8(33.4)$ & $62(20 ; 176)$ & \\
\hline \multirow[t]{2}{*}{ Gender } & Women & $34.2(30.8)$ & $25(12 ; 170)$ & 0.278 \\
\hline & Men & $38.3(28.9)$ & $24(12 ; 146)$ & \\
\hline \multirow[t]{2}{*}{ Age (years) } & $\geq 25$ & $42.5(34.5)$ & $27(12 ; 170)$ & 0.2 \\
\hline & $<25$ & $32.1(22.6)$ & $23(12 ; 115)$ & \\
\hline \multirow[t]{2}{*}{ Obesity (BMI $\geq 25 \mathrm{~kg} / \mathrm{m}^{2}$ ) } & Yes & $45.6(35.8)$ & $34(13 ; 170)$ & 0.046 \\
\hline & No & $33.8(26.1)$ & $22(12 ; 146)$ & \\
\hline \multirow[t]{2}{*}{ Smoking } & Yes & $41.1(30.1)$ & $30(13 ; 146)$ & 0.094 \\
\hline & No & $33.1(29.7)$ & $22(12 ; 170)$ & \\
\hline \multirow[t]{4}{*}{ Type of PD } & 1 & $36.0(33.1)$ & $20(13 ; 103)$ & 0.534 \\
\hline & 3 & $37.6(30.3)$ & $26(12 ; 170)$ & \\
\hline & 4 & $30.4(24.9)$ & $20(12 ; 99)$ & \\
\hline & 5 & $40.9(26.3)$ & $37(13 ; 90)$ & \\
\hline \multirow[t]{2}{*}{ Use of antibiotic prophylaxis } & Yes & $33.5(28.0)$ & $20.5(12 ; 170)$ & 0.001 \\
\hline & No & $50.1(30.9)$ & $48(12 ; 146)$ & \\
\hline \multirow[t]{2}{*}{ Use of suction drain } & Yes & $37.9(32.2)$ & $21(13 ; 170)$ & 0.739 \\
\hline & No & $36.1(24.3)$ & $27(12 ; 115)$ & \\
\hline \multirow[t]{2}{*}{ Use of methylene blue } & Yes & $36.8(27.8)$ & $25(12 ; 146)$ & 0.733 \\
\hline & No & $38.2(33.5)$ & $21(12 ; 170)$ & \\
\hline
\end{tabular}

Our finding that SSI prolongs healing time may appear intuitive but the magnitude of this effect is noteworthy: median healing time was three times longer when SSI occurred and corresponded to the duration of open wound healing reported in the literature [2]. Based on multivariable analysis, we concluded that in case of infection, patients 25 years and older had significantly longer healing times than their younger counterparts. PD typically affects young men and our finding suggests that PD may be trickier to treat in older patients. Assessment of recurrence rates was beyond the scope of this study but others have suggested that besides increasing healing time, SSI after primary closure of PD predicts a higher risk of recurrence [10].

Due to its retrospective nature, our study has several flaws. We analyzed a series of potential risk factors for SSI but were unable to include others such as the size of PD, distance from the anal verge, degree of hairiness, type of professional or physical activity. Although the vast majority of patients were regularly followed at our outpatient wound clinic where hair removal and dressing changes were performed until complete healing, we were unable to assess the impact of compulsive wound hygiene retrospectively. We were unable to measure the full impact of SSI as we did not assess time off work, wound-related discomfort, or cost. Our SSI rate of $31 \%$ lies in the upper range of SSI rates reported in the literature, particularly after off-midline primary closure techniques. The aim of the study was a critical review of SSI and our diagnostic criteria for SSI may have been wider than in other studies that were more interested in reporting success rates. Certainly, the retrospective diagnosis of SSI can be subject to error but all cases of SSI fulfilled the CDC guidelines; in over $85 \%$ of SSI cases, the diagnostic criterion was removal of sutures or incision by a physician to drain pus. Another contribution to the high SSI rate may have been the exclusion of a small yet significant number of patients (14\%) lost to follow-up, as those are more likely to have healed uneventfully.

In conclusion, this study found that following excision and primary midline closure for PD, approximately onethird of patients will develop SSI, which triples woundhealing time. Tobacco smoking and lack of preoperative antibiotic prophylaxis are independent predictors of SSI. Patients older than 25 years are at increased risk of prolonged healing time if SSI occurs. Therefore, excision and primary midline closure may not be the best therapeutic option for smokers past their early $20 \mathrm{~s}$ and other techniques such as off-midline closure or no closure and treatment by secondary healing may be preferable.

Conflict of interest None.

\section{References}

1. Sondenaa K, Andersen E, Nesvik I et al (1995) Patient characteristics and symptoms in chronic pilonidal sinus disease. Int $\mathbf{J}$ Colorectal Dis 10:39-42 
2. McCallum I, King PM, Bruce J (2007) Healing by primary versus secondary intention after surgical treatment for pilonidal sinus. Cochrane Database Syst Rev 2007:CD006213

3. Al-Khayat H, Sadeq A, Groof A et al (2007) Risk factors for wound complication in pilonidal sinus procedures. J Am Coll Surg 205:439-444

4. Tezel E (2007) A new classification according to navicular area concept for sacrococcygeal pilonidal disease. Colorectal Dis 9:575-576

5. Horan TC, Andrus M, Dudeck MA (2008) CDC/NHSN surveillance definition of health care-associated infection and criteria for specific types of infection in the acute care setting. Am J Infect Control 36:23

6. Petersen S, Koch R, Stelzner S et al (2002) Primary closure techniques in chronic pilonidal sinus: a survey of the results of different surgical approaches. Dis Colon Rectum 45:1458-1467

7. Lee PJ, Raniga S, Biyani DK et al (2008) Sacrococcygeal pilonidal disease. Colorectal Dis 10:639-650 discussion 651-632

8. Cruse PJ, Foord R (1980) The epidemiology of wound infection. A 10-year prospective study of 62, 939 wounds. Surg Clin North Am 60:27-40

9. Sondenaa K, Nesvik I, Gullaksen FP et al (1995) The role of cefoxitin prophylaxis in chronic pilonidal sinus treated with excision and primary suture. J Am Coll Surg 180:157-160

10. Sondenaa K, Diab R, Nesvik I et al (2002) Influence of failure of primary wound healing on subsequent recurrence of pilonidal sinus. Combined prospective study and randomised controlled trial. Eur J Surg 168:614-618

11. Chaudhuri A, Bekdash BA, Taylor AL (2006) Single-dose metronidazole vs 5-day multi-drug antibiotic regimen in excision of pilonidal sinuses with primary closure: a prospective, randomized, double-blinded pilot study. Int J Colorectal Dis 21:688-692

12. Lundhus E, Gottrup F (1993) Outcome at three to five years of primary closure of perianal and pilonidal abscess. A randomised, double-blind clinical trial with a complete three-year followup of one compared with four days' treatment with ampicillin and metronidazole. Eur J Surg 159:555-558
13. Cayci C, Russo M, Cheema F et al (2008) Risk analysis of deep sternal wound infections and their impact on long-term survival: a propensity analysis. Ann Plast Surg 61:294-301

14. Veeravagu A, Patil CG, Lad SP et al (2009) Risk factors for postoperative spinal wound infections after spinal decompression and fusion surgeries. Spine (Phila Pa 1976) 34:1869-1872

15. Knobloch K, Gohritz A, Reuss E et al (2008) Nicotine in plastic surgery: a review. Chirurg 79:956-962

16. Neumayer L, Hosokawa P, Itani K et al (2007) Multivariable predictors of postoperative surgical site infection after general and vascular surgery: results from the patient safety in surgery study. J Am Coll Surg 204:1178-1187

17. Jensen JA, Goodson WH, Hopf HW et al (1991) Cigarette smoking decreases tissue oxygen. Arch Surg 126:1131-1134

18. Bagaitkar J, Demuth DR, Scott DA (2008) Tobacco use increases susceptibility to bacterial infection. Tob Induc Dis 4:12

19. Buimer MG, Wobbes T, Klinkenbijl JH (2009) Hidradenitis suppurativa. Br J Surg 96:350-360

20. Anaya DA, Dellinger EP (2006) The obese surgical patient: a susceptible host for infection. Surg Infect (Larchmt) 7:473-480

21. Colak T, Turkmenoglu O, Dag A et al (2010) A randomized clinical study evaluating the need for drainage after Limberg flap for pilonidal sinus. J Surg Res 158(1):127-131

22. Erdem E, Sungurtekin U, Nessar M (1998) Are postoperative drains necessary with the Limberg flap for treatment of pilonidal sinus? Dis Colon Rectum 41:1427-1431

23. Gurer A, Gomceli I, Ozdogan M et al (2005) Is routine cavity drainage necessary in Karydakis flap operation? A prospective, randomized trial. Dis Colon Rectum 48:1797-1799

24. Doll D, Novotny A, Rothe R et al (2008) Methylene blue halves the long-term recurrence rate in acute pilonidal sinus disease. Int J Colorectal Dis 23:181-187

25. Yigit T, Yigitler C, Gulec B et al (2005) Do we need to use subcutaneous suture for pilonidal sinus treated with excision and simple primary closure? Acta Chir Belg 105:635-638 